$\Omega$
Chandima Divithotawela, Daniel Chambers, Peter Hopkins

D

The Prince Charles Hospital, Brisbane, Australia.

\title{
Pregnancy after lung transplant
}

\section{Case Report}

Improved survival now means that more women with lung transplants than ever before, many of whom have a diagnosis of cystic fibrosis, may be able to consider pregnancy. However, lung transplant recipients have increased risk of maternal and neonatal complications associated with pregnancy [1]. These pregnancies should be planned in advance and closely monitored. Pregnancy should be avoided for a minimum of 1-2 years after transplant to minimise episodes of acute rejection and achieve the lowest possible doses of immunosuppressive drugs. Immunosuppression with mycophenolate mofetil and mammalian target of rapamycin (mTOR) inhibitors should be avoided because of teratogenic effects. Immunosuppression based on prednisolone, azathioprine and calcineurin inhibitors should be established before conception. Vaginal delivery is encouraged due to the lower risk of infection and adverse events of general anaesthesia that may occur with caesarean delivery. Breast feeding is discouraged because of the risk of infant exposure to immunosuppressive drugs via breast milk, though remain a controversial recommendation. We present a patient who had successful pregnancy after lung transplant for cystic fibrosis.

\section{Case history}

A 36-year-old woman presented for advice because she was considering pregnancy. 14 years previously, she underwent bilateral sequential lung transplant for end-stage lung disease caused by cystic fibrosis. Her past medical history included diabetes mellitus, chronic renal impairment caused by tacrolimus-induced nephrotoxicity, previous cyclosporine-induced hepatitis, epilepsy soon after lung transplant that was well controlled on lamotrigine and Scedosporium sternal osteomyelitis. Maintenance immunosuppressive drugs were tacrolimus ( $2 \mathrm{mg}$ twice daily) and prednisolone (5 mg daily). Pulmonary function tests showed forced expiratory volume in $1 \mathrm{~s}$ (FEV1) $2.32 \mathrm{~L}$ (76\% predicted) and forced vital capacity (FVC) $2.7 \mathrm{~L}$ (69\% predicted). Physical examination was unremarkable and her blood pressure was 120/80 mmHg. Her creatinine level was $117 \mu \mathrm{mol} \cdot \mathrm{L}^{-1}$ and estimated glomerular filtration rate was $55 \mathrm{~mL} \cdot \mathrm{min}^{-1} .1 .73 \mathrm{~m}^{-2}$.

\section{Task 1}

What are the main issues that you would consider when giving advice to this patient?
Cite as: Divithotawela C, Chambers D, Hopkins P, et al. Pregnancy after lung transplant. Breathe 2015: 11: 291-295. 


\section{Answer 1}

- Timing of pregnancy

- Explain risk of maternal complications

- Explain risk of fetal complications

- Optimise immunosuppressive and prophylactic regimens

- Genetic counselling

- Good general health and stable graft function

- Optimise other underlying medical problems, especially blood pressure and diabetic control

Pre-pregnancy counselling was undertaken. Supplements were started including a multivitamin, folic acid (500 $\mu \mathrm{g}$ daily), calcium carbonate (600 mg daily) and vitamin D (1000 U daily). The immunosuppressive regimen was continued because it already was minimised with only two drugs. Azithromycin was added to prevent future chronic rejection. She received intensive endocrinology advice with the aim of achieving pre and post-prandial blood sugar level $<8 \mathrm{mmL} \cdot \mathrm{L}^{-1}$. Chronic kidney disease was managed by a renal physician. Lamotrigine was stopped after consultation with a neurologist because the potential risks of continuation exceeded the benefits. Genetic counselling was arranged with a specialist medical genetics service. The patient's partner was tested and had no cystic fibrosis gene mutations.

The patient received ovulation induction with clomiphene citrate and intrauterine insemination intermittently for 2 years. This resulted in one pregnancy that ended in miscarriage at 6 weeks. She received in vitro fertilisation (IVF) under strict supervision and had a successful pregnancy after 4 years of intermittent IVF cycles. She was treated jointly at tertiary care specialist high-risk obstetric, lung transplant, endocrinology and cystic fibrosis units

\section{Task 2}

True or False?

a. The FEV1 and FVC will not change markedly during pregnancy in lung transplant patients.

b. Preterm delivery and pre-eclampsia are more common in lung transplant patients than in the general population.

c. Caesarean is preferred to vaginal delivery in lung transplant patients.

d. Breast feeding is discouraged in post partum lung transplant patients.

e. Immunosuppressive target levels should be lowered in pregnancy transplant patients to avoid fetal toxicity. 


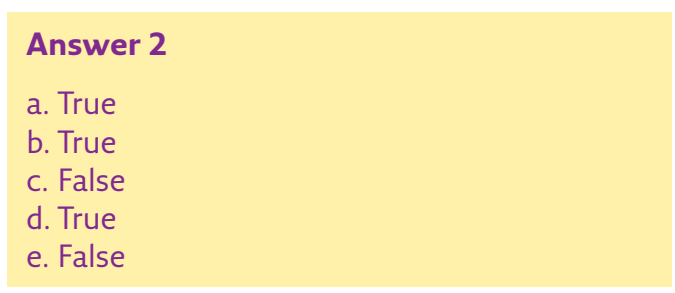

Therapeutic drug monitoring for tacrolimus was completed weekly (target $8 \mu \mathrm{g} \cdot \mathrm{L}^{-1}$ ) and the patient was followed in the clinic every 4 weeks with tests including complete blood count, serum creatinine, creatinine clearance, liver function tests, and urine microscopy, culture and protein tests. At pregnancy week 25 , an elevated eosinophil blood cell count $\left(0.55 \times 10^{9}\right.$ per $\mathrm{L})$ was noted. This raised the possibility of acute rejection. Prednisolone dose increased to $7.5 \mathrm{mg}$ and azathioprine (75 mg daily) was started. At week 29, pulmonary function deteriorated (FEV1 reduced by $190 \mathrm{~mL}$ (9\%) from baseline); bronchoalveolar lavage showed predominant lymphocytes (13\%, upper limit 7\%) and bronchial brushing showed elevated activated T-cells consistent with acute rejection. The prednisolone dose was increased to $15 \mathrm{mg}$ daily, the azathioprine dose was increased to $100 \mathrm{mg}$ daily and the tacrolimus target level was maintained at $8 \mu \mathrm{g} \cdot \mathrm{L}^{-1}$. Nebulised amphotericin (10 mg twice daily) was started for antifungal prophylaxis given the previous history of invasive fungal infection and, hence, the risk of recurrence with increased immunosuppression.

At week 32, the patient had elevated blood pressure $(150 / 90 \mathrm{mmHg})$, proteinuria (1+) and worsened renal function (creatinine $127 \mu \mathrm{mol} \cdot \mathrm{L}^{-1}$, estimated glomerular filtration rate $47 \mathrm{~mL} \cdot \mathrm{min}^{-1} \cdot 1.73 \mathrm{~m}^{-2}$ ), consistent with pre-eclampsia, and she was promptly admitted to an inpatient obstetric unit. She had emergency caesarean delivery under general and combined epidural/spinal anaesthesia, with delivery of a $2.336-\mathrm{kg}$ live boy (Apgar scores: 9 (1 $\mathrm{min})$ and $5(5 \mathrm{~min}))$. The baby was treated with continuous positive airway pressure (11 h) for mild respiratory distress and phototherapy for hyperbilirubinaemia of prematurity (maximum bilirubin level $158 \mu \mathrm{mol} \cdot \mathrm{L}^{-1}$ ). He had hypoglycaemia intermittently because of maternal diabetes and was treated with intravenous glucose. The mother had an emergency hysterectomy to control catastrophic post partum haemorrhage from a bicornuate uterus with morbidly adherent placenta.

The mother and baby were discharged to home on the 27th postoperative day. At 1 month after delivery, transbronchial lung biopsy showed no signs of acute rejection. At 2 years after delivery, the baby boy was developing normally and the mother had stable graft function with FEV $12.04 \mathrm{~L}$ (68\% predicted) (figure 1$)$.

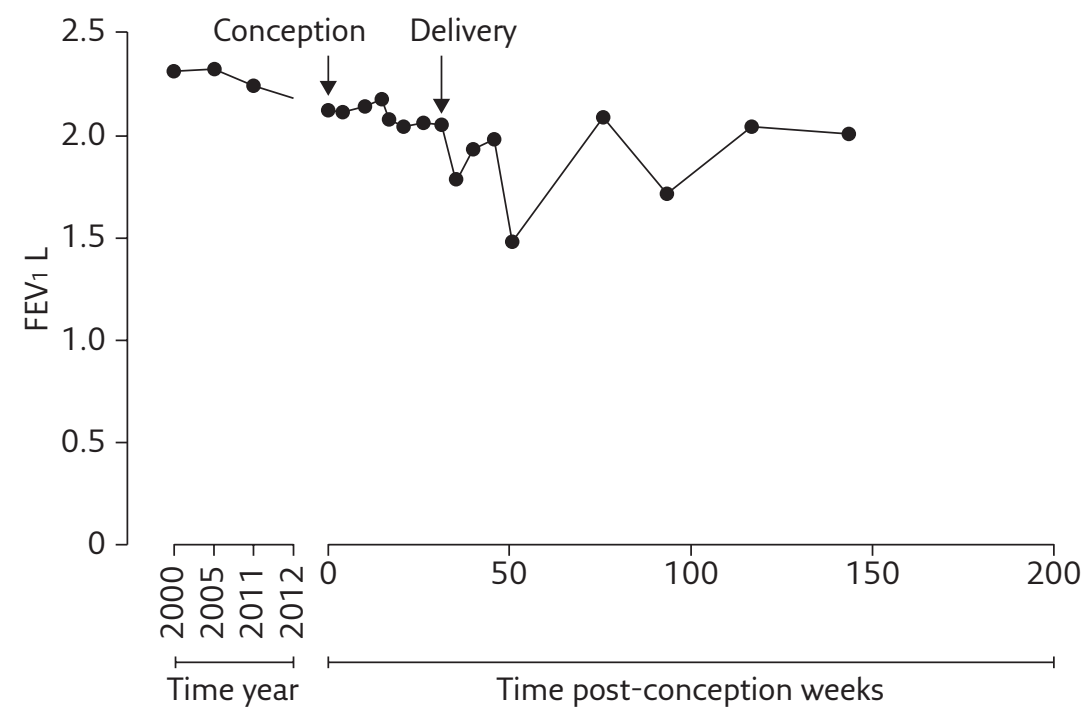

Figure 1 Forced expiratory volume in $1 \mathrm{~s}$ (FEV1) pre-and post-conception.

\section{Discussion}

Following the first report of a successful pregnancy in 1996 [2], there have been several reports of successful pregnancies after lung transplantation [1, 3-9]. Registries have been established to collect data regarding pregnancy after organ transplant, the National Transplantation Pregnancy Registry being established in the USA in 1991. In the 2010 report, there had been 30 pregnancies in 21 lung transplant recipients and five pregnancies in five heart-lung transplant recipients [10].

The first guidelines about pregnancy and transplant were published in 2005 from a consensus conference organised by the Women's Health Committee of the American Society of Transplantation [10]. The European Dialysis and Transplantation Association published recommendations about conception in renal recipients that may be extrapolated to other solid-organ transplant recipients (table 1).

\section{Timing of pregnancy}

Pregnancy should be avoided for a minimum of 1-2 years after transplant [1, 7, 11, 12]. This may enable time to decrease immunosuppression to the lowest possible maintenance doses, adequately control concomitant medical conditions, and minimise the risk of acute rejection and opportunistic infections. Adequate and stable graft function before pregnancy is recommended [11].

\section{Assisted reproductive technologies}

Reproductive ability is impaired in patients after transplant compared with the general population. Some case series have reported that $21 \%$ of patients require medication or assisted reproductive technology to achieve conception [1, 7]. This percentage is typically higher in the cystic fibrosis cohort due to the additional impact of 
Table 1 Pre-pregnancy treatment

Delay conception minimum 1 year (preferably 2 years) after transplant

Good general health and stable graft function (organ-specific assessment)

No recent episodes of acute rejection or evidence of ongoing chronic rejection

No recent episodes of infection or evidence of ongoing chronic infection (HIV, hepatitis B virus, hepatitis C virus, cytomegalovirus, herpes simplex virus, human papillomavirus, syphilis, rubella, varicella or toxoplasmosis)

Vaccinations should be given if needed (rubella, tetanus, hepatitis B virus, influenza and pneumococcus)

Normal blood pressure or mild arterial hypertension with minimal antihypertensive regimen (preferably only 1 drug; no

angiotensin-converting enzyme inhibitors)

Acceptable renal function (creatinine level $<1.5 \mathrm{mg} \cdot \mathrm{dL}^{-1}$ )

Absent or minimal proteinuria $\left(<0.5 \mathrm{~g}^{\left.-\mathrm{day}^{-1}\right)}\right.$

Absent or adequately controlled diabetes mellitus

Immunosuppressive regimen including prednisone $\left(<15 \mathrm{mg} \cdot \mathrm{day}^{-1}\right)$, azathioprine $\left(<2 \mathrm{mg} \cdot \mathrm{kg}^{-1} \cdot \mathrm{day}^{-1}\right)$, cyclosporine or tacrolimus (low therapeutic levels); mycophenolate mofetil, sirolimus and everolimus are contraindicated (and should be stopped 6 weeks before conception)

Contraception is needed until planned pregnancy

Aetiology of original disease and relevant genetic issues discussed

Risks of intrauterine growth restriction, prematurity and low birth weight discussed.

Risks of maternal complications (hypertension, pre-eclampsia, diabetes, graft rejection and graft loss) discussed

Reproduced and modified from [1] with permission from the publisher.

poor weight, cystic fibrosis-related diabetes and changes to the cervical mucus during ovulation. It is important to avoid unnecessary complications such as ovarian hyperstimulation syndrome and achieve a singleton pregnancy.

\section{Immunosuppression and other post-transplant} medications during pregnancy

The combination of immunosuppressive drugs such as a nucleotide-blocking agent (azathioprine or mycophenolate mofetil), a corticosteroid and a calcineurin inhibitor (cyclosporine or tacrolimus) may be used after transplant to enable a synergistic effect and avoid drug toxicity [1].

\section{Corticosteroids}

Prednisolone and methylprednisolone are metabolised by the placenta before reaching the fetus. No increased rate of congenital malformations has been reported in women treated with steroids during pregnancy [1]. However, steroid use during pregnancy is associated with increased incidences of diabetes, hypertension, intrauterine growth restriction and premature rupture of membranes [1].

\section{Antimetabolite drugs}

Mycophenolate mofetil should be avoided during pregnancy. Azathioprine is preferred to mycophenolate mofetil because the latter drug is associated with a high risk of structural malformations and miscarriage $[1,7]$.

\section{Calcineurin inhibitors}

The frequency of congenital malformations after exposure to cyclosporine and tacrolimus in utero is similar to that observed in the general population (5\%) [1]. Maternal complications associated with calcineurin inhibitors include increased incidences of gestational diabetes, hypertension, preterm delivery and low birth weight. Many (50\%) infants are delivered at gestation $<37$ weeks and have birth weight $<2500 \mathrm{~g}$.

\section{mTOR inhibitors}

Sirolimus and everolimus should be discontinued before pregnancy due its known teratogenic effects.

During pregnancy, plasma volume and renal blood flow are increased, enhancing the volume of distribution, causing decreased levels of immunosuppressants, especially steroids, cyclosporine and tacrolimus [7]. Reduced drug absorption due to nausea and vomiting in the first trimester and slow gut motility also may affect therapeutic drug levels. It is important to monitor therapeutic drug levels and adjust doses as needed. The present patient's tacrolimus dose was increased from $2 \mathrm{mg}$ twice daily before pregnancy to $4 \mathrm{mg}$ twice daily during pregnancy to attain the same trough level.

Macrolides are used after transplant to reduce or treat chronic rejection. Macrolides may prolong the QT interval and cause fatal torsades de pointes in the fetus. Azithromycin is a US Food and Drug Administration (FDA) category B drug 
that should be stopped or used with caution during pregnancy [1].

Other drugs such as voriconazole (FDA category D: evidence of fetal risk), valganciclovir (FDA category C: fetal risk cannot be ruled out) and ganciclovir (FDA category $C$ ) should be used when the potential benefits exceed the risks.

\section{Lung function in pregnancy}

In pregnancy, functional residual capacity is reduced (20\%), tidal volume and minute ventilation are increased, and respiratory rate is unchanged. The FEV 1 and FVC usually are unchanged [1, 7]. Any change in FEV1 should trigger investigation to rule out rejection or infection.

\section{Graft rejection and loss}

According to National Transplantation Pregnancy Registry data on solid-organ transplant recipients, there is no increased risk of rejection in patients who have stable graft function [10]. However, acute rejection incidence is higher in pregnant women who have lung than other organ transplants (lung, 36\%; heart, 20\%; liver, 10\%; kidney, $9 \%$ ) [10]. In one report, pulmonary graft loss occurred in $21 \%$ of patients within 2 years post partum [12].

\section{Effects on the fetus}

The frequency of live birth after lung transplant is $50-60 \%[1,4,7,12]$, with a relatively high risk of spontaneous or therapeutic abortion [12]. However, the frequency of congenital malformation for pregnancies in women with a history of sol- id-organ transplant is similar to that of the general population (3-4\%) [1].

The altered immune function of infants exposed to immunosuppressive drugs during pregnancy normalises by age 1 year [1]. Preterm delivery is common; in one case series of 21 patients who had pregnancy after lung transplant, $61 \%$ of deliveries occurred before 37 weeks and $61 \%$ babies had mean birth weight $<2500 \mathrm{~g}$ [3]. Breast feeding should be avoided because of passage of maternal immunosuppressive drugs in breast milk [1].

\section{Effects on the mother}

There is a high risk of developing hypertension and renal dysfunction after lung transplant. Hypertension occurs in $51 \%$ of patients within 1 year and $85 \%$ of patients within 5 years [11]. Renal dysfunction occurs in $25 \%$ of patients after 1 year and $39 \%$ of patients after 5 years [12]. Such impairment increases the risk of hypertension, pre-eclampsia and pre-term delivery. The incidence of pre-eclampsia is greater in lung transplant recipients $(6-13 \%)$ than in the general population $(2-7 \%)[1,7]$. New onset of diabetes during pregnancy is increased in lung transplant recipients (National Transplantation Pregnancy Registry: 21-23\%) [10].

Vaginal delivery is recommended to minimise blood loss and infection risk unless caesarean delivery is indicated because of obstetric problems such as pre-eclampsia [1]. Epidural anaesthesia is preferred over general anaesthesia to avoid the risks of intubation, mechanical ventilation and drug interactions [1]. Close monitoring of immunosuppression is necessary because of post partum changes in pharmacodynamics.

\section{Conflict of interest}

None declared.

\section{References}

1. Vos R, Ruttens D, Verleden SE, et al. Pregnancy after heart and lung transplantation. Best Pract Res Clin Obstet Gynaecol 2014; 28: 1146-1162.

2. Parry D, Hextall A, Robinson VP, et al. Pregnancy following a single lung transplant. Thorax 1996; 51: 1162-1164.

3. Shaner J, Coscia LA, Constantinescu S, et al. Pregnancy after lung transplant. Prog Transplant 2012; 22: 134-140.

4. Gertner G, Coscia L, McGrory C, et al. Pregnancy in lung transplant recipients. Prog Transplant 2000; 10: 109-112.

5. Kruszka SJ, Gherman RB. Successful pregnancy outcome in a lung transplant recipient with tacrolimus immunosuppression. A case report. J Reprod Med 2002; 47: 60-62.

6. Cowan SW, Coscia LC, Philips L, et al. Pregnancy outcomes in female heart and heart-lung transplant recipients. Transplant Proc 2002; 34: 1855-1856.

7. Thakrar MV, Morley K, Lordan JL, et al. Pregnancy after lung and heart-lung transplantation. J Heart Lung Transplant 2014; 33: 593-598.
8. Gyi KM, Hodson ME, Yacoub MY. Pregnancy in cystic fibrosis lung transplant recipients: case series and review. J Cyst Fibros 2006; 5: 171-175.

9. Huang JQ, Shahine LK, Gupta N, et al. Controlled ovarian hyperstimulation and gestational surrogacy in a patient with lung transplant: a case report. J Reprod Med 2010; 55 : 509-510.

10. Coscia LA, Constantinescu S, Moritz MJ, et al. Report from the National Transplantation Pregnancy Registry (NTPR): outcomes of pregnancy after transplantation. Clin Transpl 2010; 65-85.

11. McKay DB, Josephson MA, Armenti VT, et al. Reproduction and transplantation: report on the AST Consensus Conference on Reproductive Issues and Transplantation. Am J Transplant 2005; 5: 1592-1599.

12. Wu DW, Wilt J, Restaino S. Pregnancy after thoracic organ transplantation. Semin Perinatol 2007; 31 : 354-362. 